\title{
Association between abdominal obesity and subclinical hypothyroidism.
}

\author{
Santhoshakumari TMJ' ${ }^{*}$, Sneha $\mathbf{M}^{2}$ \\ ${ }^{1}$ Department of Biochemistry, Sri Lakshmi Narayana Institute of Medical Sciences, Puducherry, India \\ ${ }^{2}$ Sri Manakulavinayagar Medical College Hospital and Research Centre, Madagadipet, Puducherry, India
}

\begin{abstract}
Background: Abdominal obesity or increase in waist circumference is one of the important components of metabolic syndrome. In this study the association between waist circumference (abdominal obesity) with subclinical hypothyroidism (SCH) was analysed. Objective: To identify patients with and without abdominal obesity. To assess the association between subclinical hypothyroidism and abdominal obesity in these two groups.

Methodology: 75 subjects with abdominal obesity and 75 with normal Waist circumference (WC) were enrolled and BMI, Blood pressure (BP) and serum free T3, free T4, and TSH were done. Statistical analysis: Base line characteristics of the study participants are expressed as Mean \pm SD and percentage. The difference in anthropometric and biochemical parameters in the study group and control group was analysed by student' $t$ ' test. Results: In the study group 55 subjects $(\mathbf{7 3 \%})$ were euthyroid, 20 subjects (27\%) had subclinical hypothyroidism. Interestingly in the control group 66 subjects $(88 \%)$ were euthyroid, 9 subjects $(12 \%)$ had subclinical hypothyroidism. This shows that in our study abdominal obesity and SCH have statistically significant association.
\end{abstract}

Keywords: Abdominal obesity, Metabolic syndrome, Subclinical hypothyroidism, Infertility, BMI.

Accepted on July 11, 2019

\section{Introduction}

Metabolic syndrome (MetS) includes a cluster of risk factors like abdominal obesity, hypertension, dyslipidemia, hyperglycemia, prothrombotic and proinflammatory conditions which accelerates atherogenic process in the body $[1,2]$. Increase in waist circumference or abdominal obesity is an important marker of visceral obesity that is more dangerous than central obesity. Genetic and environmental factors play a major role in the development of obesity, metabolic syndrome and thyroid dysfunction. The National cholesterol Education programme Expert panel (NCEP EP ATP III) defines five important components of metabolic syndrome of which we have taken two components namely waist circumference and blood pressure [3] in our study.

1. Waist circumference $(>90 \mathrm{~cm}$ in male and $>80 \mathrm{~cm}$ in female)

2. Hypertension (Systolic blood pressure $>130 \mathrm{mmHg}$ and

Diastolic blood pressure $>85 \mathrm{~mm} \mathrm{Hg}$ ).

Asian population have a predisposition to abdominal obesity [4] which is one of the main components of metabolic syndrome. Various studies indicate high prevalence of metabolic syndrome in India and other South Asian countries. Serum TSH has been associated with components of metabolic syndrome. Subclinical hypothyroidism is defined as TSH level greater than $4.2 \mathrm{mU} / \mathrm{L}$ but less than $10 \mathrm{mU} / \mathrm{L}$. In various studies it was documented that subjects with subclinical hypothyroidism have a greater prevalence of high blood pressure and dyslipidemia [5,6]. In addition, obesity was significantly correlated with serum TSH [7]. Subclinical hypothyroidism is more common among obese male and female population which is not given much significance. $\mathrm{SCH}$ and increased waist circumference are closely associated with anovulatory cycles and infertility in female subjects.

Hence the study of thyroid dysfunction (TD) in population with abdominal obesity may help us to know the magnitude of overlap of these two groups and may highlight the importance of thyroid function tests in these persons.

\section{Aim and Objective}

- To identify patients with and without abdominal obesity.

- To do thyroid function tests in subjects with and without abdominal obesity.

- To investigate the association between subclinical hypothyroidism and abdominal obesity.

\section{Review Literature}

In studies on subjects with subclinical hypothyroidism, it was found that they had a higher prevalence of high blood pressure and dyslipidemia [5,8]. In addition obesity, especially abdominal obesity was significantly correlated with TSH [9]. 
In a study with a follow up period of 3 years it was found that subjects with metabolic syndrome had an increase in TSH over time [10]. Subclinical hypothyroidism with a TSH between 4.2-10 mU/L was significantly associated with prevalence of metabolic syndrome [11]. Low thyroid hormone level is associated with occurrence of obesity and hence can contribute to the development of metabolic syndrome [12]. The Jaipur Heart Watch Studies in urban Indian populations, the prevalence of metabolic syndrome was much higher in women $(30.9 \%)$ [13].

Sub-clinical hypothyroidism ( $\mathrm{SCH}$ ) and overt hypothyroidism are recognized risk factors for atherosclerotic cardiovascular disease, hyperlipidemia, low grade inflammation and hypercoagulability $[14,15]$. Thyroid hormones affect lipid metabolism, carbohydrate metabolism, blood pressure and energy homeostasis $[16,17]$. Studies have given evidence that there may be some association between impaired thyroid function and components of MetS [5]. A cross-sectional study on South Indian patients demonstrated an association between hypothyroidism (overt and sub-clinical) and MetS and showed that women with MetS are at greater risk of developing hypothyroidism [18].

\section{Materials and Methods}

\section{Materials}

75 patients with abdominal obesity as per the NCEP ATP III criteria and 75 controls attending the OP clinic of Sri Lakshmi Narayana Institute of Medical Sciences were selected as subjects for this study.

\section{Inclusion criteria}

Obese and non-obese individuals.

\section{Exclusion criteria}

Known cases of hypothyroidism.

\section{Methods}

Waist circumference was measured using stretch resistant tape. Blood pressure was measured using digital BP monitor
(CH-432, CITIZEN Microhuman Tech) $5 \mathrm{ml}$ of venous blood was collected in vacutainers from which serum was separated by centrifuging at $3000 \mathrm{rpm} /$ minute. Serum free T3, free T4, and TSH by Enzyme linked immunosorbent assay technique (ELISA).

\section{Statistical Analysis}

Base line characteristics of the study participants are expressed as Mean \pm SD and percentage. The difference in anthropometric and biochemical parameters in the study group and control group was analysed by student ' $t$ ' test. Statistical analysis was performed using SPSS window version 17.

Table 1: Anthropometric and blood pressure measurements of the study and control group. *: Significant Values.

\begin{tabular}{llll}
\hline \multirow{2}{*}{ Parameters } & Control group & Study group & p value \\
\cline { 2 - 4 } & $(\mathbf{n}=\mathbf{7 5 )}$ & $(\mathbf{n}=\mathbf{7 5})$ & \\
\hline BMI & $22.35 \pm 2.41$ & $27.86 \pm 1.7$ & $0.001^{*}$ \\
\hline Waist circumference & $79.9 \pm 5.5$ & $93.2 \pm 5.7$ & $0.000^{*}$ \\
\hline Systolic BP & $113.5 \pm 11.4$ & $128.1 \pm 13.8$ & $0.000^{*}$ \\
\hline Diastolic BP & $73.48 \pm 5.61$ & $77.57 \pm 6.41$ & $0.000^{*}$ \\
\hline
\end{tabular}

There was significantly higher BMI, waist circumference, systolic and diastolic blood pressure in the study group as compared to the control group (Table 1).

Table 2: Biochemical parameters between study group and control group.

\begin{tabular}{llll}
\hline Parameters & Control group $(\mathbf{n}=\mathbf{7 5})$ & Study group $(\mathbf{n}=\mathbf{7 5})$ & p value \\
\hline Free T3 & $2.07 \pm 1.04$ & $1.74 \pm 0.76$ & $0.031^{*}$ \\
\hline Free T4 & $1.6 \pm 0.63$ & $1.3 \pm 0.54$ & $0.002^{*}$ \\
\hline TSH & $3.15 \pm 4.5$ & $12.48 \pm 8.16$ & $0.005^{*}$ \\
\hline
\end{tabular}

The biochemical parameters like free T3, free T4 and TSH was significantly higher in the study group as compared to the control group (Table 2).

Table 3: Shows the number and percentage of subjects with Euthyroidism and Subclinical Hypothyroidism in both the Study and Control group.

\begin{tabular}{|c|c|c|c|}
\hline TSH levels in $\mu \mathrm{IU} / \mathrm{I}$ & Control group (no:/\%) & Study group (no:/\%) & \\
\hline Euthyroid (0.46-4.2) & $66(88)$ & $55(73)$ & Chisquare Test $=5.172$ \\
\hline Subclinical Hypothyroidism (4.2-10) & $9(12)$ & $20(27)$ & $p$ value $=0.019^{*}$ \\
\hline
\end{tabular}

From the above statistics it is evident that there is significant $\mathrm{p}$ value of 0.019 which proves that there is definite association between abdominal obesity and subclinical hypothyroidism (Table 3).

\section{Discussion}

Abdominal obesity (waist circumference) an important component of metabolic syndrome was significantly high in the study group. In the study group 55 subjects (73\%) were euthyroid, 20 subjects $(27 \%)$ had subclinical hypothyroidism. Interestingly in the control group 66 subjects (88\%) were 
euthyroid, 9 subjects (12\%) had subclinical hypothyroidism. A study by Meher et al. [15] showed a high prevalence of SCH $(22 \%)$ in the MetS group. A study by Shantha et al. [16] in India has shown a high prevalence of $\mathrm{SCH}(21.90 \%)$ in patients with MetS. In our study $27 \%$ had subclinical hypothyroidism. Yet, another study in Taiwan by Lai et al. [5] showed a prevalence of subclinical hypothyroidism $(5.50 \%)$. This is very low compared to our study. Another study by Uzunlulu et al. [19] in Turkey had shown SCH prevalence to be $16.40 \%$ in the MetS group which is also low compared to our study.

In contrast to our finding a study in India states that of the seventy six patients with MetS, $53.0 \%$ to have $\mathrm{SCH}$, which is almost double the percentage observed in our study. Similarly, Jayakumar et al. [20] found that out of 120 patients who were diagnosed to have MetS, thyroid functions were normal in 50 patients, 52 had $\mathrm{SCH}$. In our study, in the study group $36.4 \%$ female and $13 \%$ male had thyroid disorder and in the control group $11.5 \%$ females had thyroid disorder. This is similar to a latest study that revealed that the prevalence of TD was more among the females with MetS as compared to male subjects, which is consistent with previous reports [21-25]. As suggested in various studies the percentage of female subjects with metabolic syndrome and associated thyroid disorder is significantly high in our study.

There are few limitations of the present study, first is that; this is a cross-sectional study, a cause and effect of relationship could not be determined. Therefore, large epidemiological studies are needed to evaluate the relationship between subclinical hypothyroidism and abdominal obesity.

\section{Conclusion}

Since the prevalence of subclinical hypothyroidism is more among subjects with abdominal obesity, more so in female, early detection and thyroxine replacement could reduce the significant cardiovascular risk and fertility related problems in these patients.

\section{References}

1. Eckel RH,Grundy SM, Zimmer PZ. The metabolic syndrome. Lancert 2005; 365: 1425-28.

2. Grundy SM. Metabolic syndrome connecting and reconciling Cardiovascular and diabetes worlds. J Am Coll Cardiol 2006; 47: 1093-1100.

3. Executive summary of the third report of the NCEP EP on detection and Evaluation of treatment of high blood cholesterol in adults. JAMA 2001; 285: 2486-2497.

4. Reaven GM, Banting lecture 1988. Role of insulin resistance in human disease. Diabetes 1988; 37: 1595-1607.

5. Lai Y, Wang J, Jiang F, Wang B, Chen Y, Li M, Liu H, Li C, Xue H, Li N, Yn J, Shi L, Bai X, Hou X, Zhu L, Lu L, Wang S, Xing Q, Teng X, Teng W, Shan Z. The relationship between serum thyrotropin and components of metabolic syndrome. Endocr J 2011; 58: 23-30.
6. Li Lu, Beibei Wang, Zhongyan Shan, Fengwei Jiang, Xiaochun Teng, Yanyan Chen, Yaxin Lai, Jiani Wang, Haibo Xue, Sen Wang,Chenyan Li, He Liu, Ningna Li, Jiashu Yu, Liangfeng Shi, Xin Hou, Qian Xing, Xue Bai, Weiping Teng. The correlation between Thyrotropin and dyslipidemia in population based study. J Korean Med Sci 2011; 26: 243-249.

7. Åsvold BO, Vatten LJ, Nilsen TIL, Bjør T. The hunt study. Eur J Endocrinol 2007; 156: 181-186.

8. Park SB, Choi HC, Joo NS. The relation of thyroid function to components of metabolic syndrome in Korean men and women. Journal of Korean Medical Science 2011; 26: 540-545.

9. American Association of Clinical Endocrinologists medical guidelines for clinical practice for the evaluation and treatment of hyperthyroidism and hypothyroidism. Endocr Pract 2002; 8: 457-69.

10. Asvold BO, Vatten LJ, Nilsen TI, Bjoro T. The association between TSH within the reference range and serum lipid concentrations in apopulation-based study. The HUNT Study Eur J Endocrinol 2007; 156: 707.

11. Pearce EN. Hypothyroidism and dyslipidemia: Modern concepts and approaches. Curr Cardiol Rep 2004; 6: 451-456.

12. Tuzcu A, Bahceci M, Gokalp D, Tuzun Y, Gunes K. Subclinical hypothyroidism may be associated with elevated high-sensitive c-reactive protein (low grade inflammation) and fasting hyperinsulinemia. Endocr $\mathrm{J}$ 2005; 52: 89-94.

13. Serter R, Demirbas B, Culha C, Cakal E. The effect of Lthyroxine replacement therapy on lipid based cardiovascular risk in sub clinical hypothyroidism. Invest $\mathbf{J}$ Endocrinol 2004; 27: 897-903.

14. American Association of Clinical Endocrinologists medical guidelines for clinical practice for the evaluation and treatment of hyperthyroidism and hypothyroidism. Endocr Pract 2002; 8: 457-69.

15. Meher LK, Raveendranathan SK, Kota SK, Sarangi J, Jali SN. Prevalence of hypothyroidism in patients with metabolic syndrome. Thyroid Res Pract 2013; 10: 60-64.

16. Shantha GP, Kumar AA, Jeyachandran V, Rajamanickam D, Rajkumar K, Salim S, Subramanian KK, Natesan S. Association between primary hypothyroidism and metabolic syndrome and the role of $\mathrm{C}$ reactive protein: a cross-sectional study form South India. Thyroid Res 2009; 2: 2 .

17. Ayturk S, Gursoy A, Kut A, Anil C, Nar A, Tutuncu NB. Metabolic syndrome and its components are associated with increased thyroid volume and nodule prevalence in a mild-to-moderate iodine-deficient area. Eur J Endocrinol 2009; 161: 599-605.

18. Tarcin O, Abanonu GB, Yazicin O. Association of metabolic syndrome parameters with TT3 and FT3/Ft4 ratio in obese Turkish population. Metabolic Syndrome Related Disorders 2012; 10: 137-142. 
19. Uzunlulu M, Yorulmaz E, Oguz A. prevalence of subclinical hypothyroidism in patients with metabolic syndrome. Endocr J 2007; 54: 71-76.

20. Jayakumar RV. Hypothyroidism and metabolic syndrome. Thyroid Res Pract 2013; 10: 1-2.

21. Garduno-Garcia Jde J, Alvirde-Garcia U, Lopez-Carrasco G, Padilla Mendoza ME, Mehta R, Arellano-Campos O, Choza R, SauqueL, Garay-Sevilla ME, Malacara JM, Gomez-Perez FJ, Aguilar-Salinas CA. TSH and free thyroxine concentrations are associated with differing metabolic markers in euthyroid subjects. Eur J Endocrinol 2010; 163; 273-278.

22. Singh BM, Goswami B, Mallika V. Association between insulin resistance and hypothyroidism in females attending a tertiary care hospital. Indian J Clin Biochem 2010; 25; 141-145.

23. Wang JY, Wang CY, Pei D, Lai CC, Chen YL, Wu CZ, Chang YL, Hsu CH, Pei C, Tang SH. Association between thyroid functions and metabolic syndrome in elderly subjects. J Am Geriatr Soc 2010; 58: 1613-1614.
24. Tehrani FR, Tohidi M, Dovom MR, Azizi F. A population based study on the association of thyroid status with components of the metabolic syndrome. J Diabetes Metab 2011; 2: 156-162.

25. Lai CC, Tang SH, Pei D, Wang CY, Chen YL, Wu CZ, Hsiao FC, Chen HS; Wang JY. The prevalence of subclinical thyroid dysfunction and its association with metabolic syndrome in Taiwanese elderly. Int $\mathrm{J}$ Gerontol 2011; 5: 25-29.

\section{*Correspondence to}

Santhoshakumari TMJ

Department of Biochemistry

Sri Lakshmi Narayana Institute of Medical Sciences

Puducherry

India 